\title{
Can a Neurosteroid Ameliorate Fragile X-Associated Tremor/Ataxia Syndrome?
}

\author{
Dejan B. Budimirovic ${ }^{1}$
}

Published online: 7 September 2017

(C) The American Society for Experimental NeuroTherapeutics, Inc. 2017

Fragile X-associated tremor/ataxia syndrome (FXTAS) is a progressive neurodegenerative disorder that affects approximately $45 \%$ of male and $16 \%$ of female carriers of a fragile $\mathrm{X}$ mental retardation 1 (FMR1) gene premutation over the age of 50 years [1]. Premutations of 55 to 200 CGG repeats of the FMR1 gene translate into increased FMR1 mRNA, inducing a toxic gain of function and/or translation of CGG repeats into a polyglycine-containing protein, FMRpolyG [1,2]. Notably, almost all premutation carriers with neurological symptoms present with FXTAS and/or have inclusions on autopsy [3]. Such pathology includes eosinophilic ubiquitin-positive intranuclear inclusions, which contain FMR1 mRNA and numerous proteins in neurons and astrocytes [4-6]. Defining clinical features of FXTAS include progressive action tremor, gait ataxia, impaired executive function and memory deficits, peripheral neuropathy, and parkinsonism [1]. Associated variable features include cognitive impairment and dementia; psychiatric symptoms, such as depression; and dysautonomia [7-9]. Radiologically, FXTAS is characterized by progressive global brain atrophy and white matter disease of both the cerebellum and cerebrum $[10,11]$. Importantly, cerebellar and brainstem atrophy and ventricular enlargement are detectable even before the onset of tremor or ataxia $[12,13]$. Currently, no specific treatment for FXTAS can slow the progression of neurodegeneration, though symptomatic treatments exist for some of the symptoms, such as tremor [14]. Only one previous controlled trial has been performed in

Dejan B. Budimirovic dbudimi1@jhu.edu

1 Departments of Psychiatry and Behavioral Sciences, Kennedy Krieger Institute and Child Psychiatry, The Johns Hopkins Medical Institutions, Johns Hopkins University School of Medicine, Baltimore, MD, USA individuals with FXTAS, a memantine trial lasting 1 year, but this treatment did not improve tremor, ataxia, or executive function $[15,16]$.

The study in this issue of Neurotherapeutics by Wang et al. [17] assessed whether allopregnanolone, a neurosteroid that promotes regeneration and repair, can improve clinical symptoms, brain activity, and magnetic resonance imaging (MRI) measurements in patients with FXTAS. In this preliminary, 3month, open-label, uncontrolled trial, 6 men with FXTAS underwent weekly intravenous infusions of allopregnanolone ( $26 \mathrm{mg}$ over $30 \mathrm{~min}$ ) for 12 weeks. The Richmond Agitation Sedation Scale was administered before, during, and after each infusion. Plasma allopregnanolone levels were determined by liquid chromatography-tandem mass spectrometry in 1 subject from blood samples drawn at intervals during and after a 6-mg infusion from the opposite arm of that receiving the intravenous infusion.

All patients completed baseline and follow-up clinical examinations and neuropsychological assessments. Functional outcomes included quantitative measurements of tremor and ataxia, as well as neuropsychological evaluations. In line with recent recommendations in the fragile $\mathrm{X}$ field [18], a wide range of neuropsychological, behavioral, neurophysiological, and structural imaging studies were applied. For example, learning and memory was measured via the California Verbal Learning Test 2, whereas working memory was assessed using Wechsler Memory Scale-IV. Measuring of brain activity consisted of event-related potential (ERP) N400 word repetition effect during a semantic memory processing task. Structural MRI outcomes comprised volumes of the hippocampus, amygdala, and fluid-attenuated inversion recovery hyperintensities, and microstructural integrity of the corpus callosum. It is noteworthy that before the treatment, the patients exhibited impairment in executive function, verbal fluency and learning, and had progressive deterioration of all MRI measurements. 
This open-label study demonstrated that intravenous infusion of allopregnanolone at doses up to $6 \mathrm{mg}$ is well tolerated and without apparent adverse effects in men with FXTAS. The half-life of allopregnanolone following termination of the infusion was approximately $35 \mathrm{~min}$. By contrast, in a prior study, subjects were dosed with 3 successive boluses without a flush for $30 \mathrm{~min}$ following each of the boluses so that drug levels were elevated over a more prolonged period of time, increasing the likelihood that side effects would be perceived, and changes in saccadic eye movements were also consistent with sedation.

In the present study, only 1 participant, the least affected patient in this cohort, experienced subjective improvement (improved balance and restored ability to walk down stairs, and a resolution of symptoms of peripheral neuropathy, including numbness and pain). The other five participants did not experience improvement in their ataxia or balance symptoms, and none of the patients experienced improvement in their tremor. These subjective experiences are validated by the lack of significant improvement in relevant measures of tremor or balance. Nevertheless, following treatment, participants demonstrated significant improvement in some cognitive domains such as executive function, episodic memory, and learning, the most affected domains in the 6 participants at baseline and in prior studies of patients with FXTAS [19]. N400 repetition effect amplitude also increased with the treatment in this study, and in the memantine trial [16].

Brain structures that are affected in FXTAS and could potentially have shown effects of allopregnanolone were also evaluated: the hippocampus, the site of adult neurogenesis [20]; the amygdala, where neurosteroids exert anxiolytic effect [21,22]; and the corpus callosum, where the white matter structure severely damaged in FXTAS [23, 24]. Examination of serial MRI scans acquired before the treatment consistently confirmed progressive deterioration in all 3 structures across all the data points. Namely, compared with controls, patients exhibited acceleration of hippocampal atrophy, subcortical hyperintensity expansion, and structural deterioration in the corpus callosum before the treatment. After the treatment, while none of the changes in MRI measurements reached statistical significance as a group, likely owing to significant interpatient variability, both improvement and deterioration in MRI measurements occurred in individual patients posttreatment in contrast to uniform deterioration prior to the treatment. Overall, significant correlations between baseline MRI measurements and changes in neuropsychological test scores suggested the effects of allopregnanolone on improving executive function, learning, and memory for patients with relatively preserved hippocampus and corpus callosum, while reducing self-reported symptoms of anxiety for patients with small hippocampi and amygdalae.

Similar to some extent to the memantine study [16], in this study Wang et al. [17] found improvement of the N400 repetition effect amplitude, thought to be an index of semantic processing and verbal learning/memory $[16,25]$. The ERP assessments were conducted in 4 patients, all of whom demonstrated improvements in the N400 repetition effect. This result suggests that the $\mathrm{N} 400$ repetition effect, primarily originating from the temporal neocortex [26], is sensitive to memory changes in FXTAS $[16,25]$.

The study has a number of limitations. This preliminary trial was conducted to obtain evidence of treatment benefits in a small sample, and thus the study was not powered to detect statistical significance while controlling for multiple comparisons. The substantial amount of interpatient variability is likely another contributing factor to the nonsignificant results, given the small sample size. Since this was not a randomized controlled study, the possibility that the aforementioned improvements were due to a placebo effect cannot be ruled out, although the improvements seen in the ERP results, neuropsychological testing, and MRI findings suggest that allopregnanolone could have had a disease-modifying effect. Despite all the statistical analyses provided in the study, it should be noted that all analyses were based on a small sample size as to provide preliminary evidence on the clinical efficacy of allopregnanolone; thus, an extra level of caution is warranted in interpreting the results. The lack of a placebo group is an additional study limitation.

In summary, this open-label trial by Wang et al. [17] provides preliminary evidence that weekly infusions of allopregnanolone in individuals with FXTAS may improve deficits in executive function, learning, and memory. The study did not, however, show evidence of a benefit for the hallmark neurological symptoms of tremor and ataxia. Subtle improvements on some MRI measurements were also noted for some patients, suggesting that allopregnanolone could be disease-modifying after only 12 weeks of treatment, possibly through an effect on neurogenesis or via neuroprotection. These preliminary results of improved cognitive functioning in patients with FXTAS, and partial alleviation of some aspects of neurodegeneration, along with a good tolerance profile, suggest that controlled trials of allopregnanolone over a longer treatment period are warranted.

Required Author Forms Disclosure forms provided by the authors are available with the online version of this article.

\section{References}

1. Hagerman PJ, Hagerman RJ. Fragile X-associated tremor/ataxia syndrome. Ann N Y Acad Sci 2015;1338:58-70.

2. Sellier C, Buijsen RA, He F, et al. Translation of expanded CGG repeats into FM RpolyG is pathogenic and may contribute to fragile X tremor ataxia syndrome. Neuron 2017;93:331-347.

3. Martínez-Cerdeño V, Lechpammer M, Hagerman PJ, Hagerman R. Two FMR1 premutation cases without nuclear inclusions. Mov Disord 2017 Jun 1 [Epub ahead of print]. 
4. Greco CM, Berman RF, Martin RM, et al. Neuropathology of fragile X-associated tremor/ataxia syndrome (FXTAS). Brain 2006;129: 243-255.

5. Iwahashi CK, Yasui DH, An HJ, et al. Protein composition of the intranuclear inclusions of FXTAS. Brain 2006;129:256-271.

6. Ariza J, Rogers H, Monterrubio A, et al. A majority of FXTAS cases present with intranuclear inclusions within Purkinje cells. Cerebellum 2016;15:546-551.

7. Hagerman RJ, Leehey M, Heinrichs W, et al. Intention tremor, parkinsonism, and generalized brain atrophy in male carriers of fragile X. Neurology 2001;57:127-130.

8. Berry-Kravis E, Abrams L, Coffey SM, et al. Fragile X-associated tremor/ataxia syndrome: clinical features, genetics, and testing guidelines. Mov Disord 2007;22:2018-1030.

9. Tassone F, Berry-Kravis EM, editors. Fragile X-associated tremor ataxia syndrome (FXTAS). New York: Springer; 2010.

10. Jacquemont S, Hagerman RJ, Leehey M, et al. Fragile X premutation tremor/ataxia syndrome: molecular, clinical, and neuroimaging correlates. Am J Hum Genet 2003;72:869-878.

11. Hashimoto R, Javan AK, Tassone F, Hagerman RJ, Rivera SM. A voxel-based morphometry study of grey matter loss in fragile $\mathrm{X}$ associated tremor/ataxia syndrome. Brain 2011;134:863-878.

12. Cohen S, Masyn K, Adams J, et al. Molecular and imaging correlates of the fragileX-associated tremor/ataxia syndrome. Neurology 2006;67:1426-1431.

13. Wang JY, Hessl D, Hagerman RJ, et al. Abnormal trajectories in cerebellum and brainstem volumes in carriers of the fragile $\mathrm{X}$ premutation. Neurobiol Aging 2017;55:11-19.

14. Hall DA, Leehey MA, Berry-Kravis E, Hagerman RJ. Treatment and management of FXTAS. In: Tassone F, Hall DA, editors. FXTAS, FXPOI, and Other Premutation Disorders. AG Switzherland: Springer International Publishing; 2016. p. 181-198.

15. Seritan AL, Nguyen DV, Mu Y, Tassone F, et al. Memantine for fragile $\mathrm{X}$-associated tremor/ataxia syndrome: a randomized, doubleblind, placebo-controlled trial. J Clin Psychiatry 2014;75:264-271.

16. Yang JC, Niu YQ, Simon C, et al. Memantine effects on verbal memory in fragile X-associated tremor/ataxia syndrome (FXTAS): a double-blind brain potential study. Neuropsychopharmacology 2014;39: 2760-2768.

17. Wang JY, Trivedi AT, Carrillo NR, et al. Open-label allopregnanolone treatment of men with fragile $\mathrm{X}$-associated tremor/ataxia syndrome. Neurotherapeutics 2017 Jul 13 [Epub ahead of print].

18. Budimirovic DB, Berry-Kravis E, Erickson CA, et al. Updated report on tools to measure outcomes of clinical trials in fragile $\mathrm{X}$ syndrome. J Neurodev Disord 2017;9:14.

19. Grigsby J, Cornish K, Hocking D, et al. The cognitive neuropsychological phenotype of carriers of the FMR1 premutation. J Neurodev Disord 2014;6:28.

20. Lieberwirth C, Pan Y, Liu Y, Zhang Z, Wang Z. Hippocampal adult neurogenesis: Its regulation and potential role in spatial learning and memory. Brain Res 2016;1644:127-140.

21. Akwa Y, Purdy RH, Koob GF, Britton KT. The amygdala mediates the anxiolytic-like effect of the neurosteroid allopregnanolone in rat. Behav Brain Res 1999;106:119-125.

22. Wang C, Marx CE, Morrow AL, Wilson WA, Moore SD. Neurosteroid modulation of GABAergic neurotransmission in the central amygdala: a role for NMDA receptors. Neurosci Lett 2007;415:118-123.

23. Wang JY, Hessl D, Schneider A, Tassone F, Hagerman RJ, Rivera SM. Fragile Xassociated tremor/ataxia syndrome: influence of the FMR1 gene on motor fiber tracts in males with normal and premutation alleles. JAMA Neurol 2013;70:10221029.

24. Wang JY, Hessl DH, Hagerman RJ, Tassone F, Rivera SM. Agedependent structural connectivity effects in fragile $\mathrm{X}$ premutation. Arch Neurol 2012;69:482-489.

25. Yang JC, Chi L, Teichholtz S, et al. ERP abnormalities elicited by word repetition in fragile $\mathrm{X}$-associated tremor/ataxia syndrome (FXTAS) and amnestic MCI. Neuropsychologia 2014;63:34-42.

26. Halgren E, Dhond RP, Christensen N, et al. N400- like magnetoencephalography responses modulated by semantic context, word frequency, and lexical class in sentences. Neuroimage 2002;17:1101-1016. 